in Advanced Joining Technologies,

T.H. North, ed., Chapman and Hall, London, U.K., 61, 1990.

\title{
THE PHYSICS OF ARC WELDING PROCESSES
}

\section{T.W. EAGAR}

Department of Materials Science and Engineering,

Massachusetts Institute of Technology

\begin{abstract}
Welding is an extremely complex process; however, due to its commercial importance, it is essential that a more thorough study of the various processes be undertaken. Three examples of areas requiring greater understanding of arc physics are presented. The first discusses heating or cooling of the metal at the cathode; the second describes variations in heat transfer using various shielding gases and the last describes droplet formation in gas metal arc welding.

Key Words: Arc Welding, Arc Physics, Shielding Gases, Gas Metal Arc Welding.
\end{abstract}

\section{Introduction}

Langmuir, compton and cobine are but a few of a number of outstanding physicists who contributed greatly to our understanding of atmospheric pressure arcs during the first half of this century. However, during the past forty years, few physicists have studied such problems. I have been told by some physicists that arc physics is too mundane and too simple a problem to challenge a modern day physicist. This, of course, is merely an excuse. Atmospheric pressure arcs are too complex and represent too difficult a problem for these people to study. Stated simply, atmospheric pressure arcs and welding present problems which are too difficult and too complicated to be approached by the type of physicists that are educated in. today's world.

The complexity of welding is readily apparent when one considers that fusion welding involves temperature gradients of thousands of degrees, over distances of less than a centimeter, occuring on a time scale of seconds, involving multiple phases of solids, liquids, gases and plasma. This complexity causes most scientists to shun welding as unscientific and unworthy of thoughtfui analysis. It is only the true engineer who is willing to deal with a process of such exceeding complexity in order 
to achieve the end result of a fabricated product of commercial and societal usefulness. One of the challenges facing those who engineer welded structures and components, is communication of the importance and significance of welding to other scientists and to the general public. one of the purposes of this paper is to describe some of the complexity of several welding processes, and to demonstrate how study of arc physics can help provide a better understanding of arc welding processes.

\section{Anode and Cathode Spot Heating}

Cobine [1955] and others have shown that the heat transferred to the anode, $Q_{A}$, is given by

$$
Q_{A}=I\left(\phi_{A}+V_{A}+\frac{3}{2} \frac{k T}{e}\right)
$$

where: $I$ is the welding current in amperes

$\phi_{A}$ is the work function or heat of condensation of the electrons expressed in volts

$V_{A}$ is the anode voltage drop

$k$ is the Boltzmann's constant

$T$ is the absolute temperature of electrons in the plasma, and

$e$ is the electron charge

By symmetry, the heat at the cathode should be given by

$$
Q_{c}=-I\left(\phi_{c}+V_{c}+\frac{3}{2} \frac{k T}{e}\right)
$$

where the sign of the current is reversed due to reversal of electron flow. Such a simplistic formulation of cathode heating may not be valid due to the much more complex (and essentially unknown) physics which occur in the cathode spot [Ecker, 1980]. Nonetheless, the sign reversal of the current clearly indicates that the exiting electrons cool the cathode. The removal of electrons to the plasma causes a cooling of the cathode even as a vaporizing gas removes heat from a liquid.

Experimental confirmation of cathodic cooling was obtained by Ishikawa et al. [1965] who used an infrared pyrometer to show that the hottest portion of a gas tungsten welding arc is not located at the tip, but several millimeters up the electrode. When the cathode is liquid, as in gas metal arc welding, convection within the weld pool essentially negates the local cooling effect of electron vaporization. Thus, cathodic cooling is an important factor when the cathode is solid; but when the cathode is liquid, cathodic cooling has little influence on 
temperature gradients, although it may alter the overall heat balance.

Nonetheless, cathodic cooling may be important in influencing cathode spot motion on the liquid weld pool. Electron emission from the weld pool is influenced by the temperature of the cathode; a hotter cathode produces emission more readily. When gas metal arc welding of most metals, the cathode spot wanders rapidly across the liquid surface. Does it do this because cathodic cooling produces easier electron emission in the hotter areas adjacent to the cathode spot? Or does cathodic cleaning of surface active oxygen at the cathode spot create a region of easier electron emission on adjacent surface regions where a thin oxygen film remains? Does the addition of oxygen to argon when gas metal arc welding of steel produce an oxygen surface film that influences arc stability? What is the effect of metal vaporization on the properties of the plasma immediately above the cathode spot? Does this high concentration of easily ionized metal vapors create stability for the cathode spot or does it cause the spot to wander about the cathode surface? These and other questions remain unanswered, yet our process modelling tools have progressed to a sufficient level that some could be answered - at least qualitatively.

clearly, there is much to be learned about the science of atmospheric pressure arcs as they relate to welding. Some of these questions could lead to answers of commercial importance.

\section{Heat Transfer Using Various Shielding Gases}

It is well known that one will melt more metal with a 100 ampere helium arc than with a 100 ampere argon arc. The conventional wisdom says that this is due to the higher ionization potential of helium $(24.6 \mathrm{eV})$ as compared to argon $(15.8 \mathrm{eV})$. It is assumed based upon the Saha equation that this will result in a higher arc temperature. Unfortunately, based on experimental measurements [Key, 1983], there is not a higher temperature in the helium welding arc: and even if there were, it could not explain the much greater melting efficiency when using helium. The correct explanation lies in the variation of the thermal conductivity of the gases.

Although eighty percent of the heat in the argon arc is carried by the electron current, twenty percent is due to heat conductance across a gas boundary layer at the anode surface. [Quiqley et al., 1973; Dinesclu and Pfender, 1980] Heat flow across this boundary layer is controlled by the thermal conductivity of the gas. For monatomic gases such as helium and argon, the thermal conductivity is controlled by the mass diffusivity of the atoms, which, according to the kinetic theory of gases, is proportional to the inverse 
square root of the mass of the atom [Dunn and Eagar, 1986]. Thus, with an atomic weight ten times as great as helium, argon has a thermal conductivity only 30 percent as large as helium. All other factors being equal, helium will conduct three times as much heat across the boundary layer as will argon. Table 1 illustrates this point. Although only 20 percent of the heat entering the anode from the argon arc is conducted across the boundary layer, $43 \%$ of the heat in a helium arc will be conducted"across the boundary layer. (These figures are only "back of the envelope" calculations. Many other parameters change when switching from argon to helium gas. other factors such as anode voltage drop, electric current distribution, arc temperature or radiation will change, but by insignificant amounts as compared to the thermal conductivity.)

As pointed out by Giedt et al. [1989], the actual melting efficiency of the arc welding process is relatively low (i.e. on the order of 20 percent or less). Thus an increase of $40 \%$ in the heat supplied could double the volume of metal melted. This is why helium shielded arcs melt more metal than argon shielded arcs.

\begin{tabular}{|cccc|}
\hline $\begin{array}{c}\text { Shielding } \\
\text { Gas }\end{array}$ & $\begin{array}{c}\text { Electric } \\
\text { Current }\end{array}$ & $\begin{array}{c}\text { Conduction } \\
\text { across gas } \\
\text { boundary } \\
\text { layer }\end{array}$ & $\begin{array}{c}\text { Percent by } \\
\text { conduction }\end{array}$ \\
\hline \hline Argon & $80 \%$ & $20 \%$ & $20 \%$ \\
Helium & $80 \%$ & $60 \%$ & $43 \%$ \\
\hline
\end{tabular}

Table 1. Relative Heat Transfer from the Welding Plasma in Argon and Helium Shielding Gases (AIl heat values normalized to $100 \%$ for argon).

In addition to these pure gases, this hypothesis can explain why gas mixtures such as 75 Ar - 25 He or 50 Ar $50 \mathrm{He}$ will increase the rate of heat transfer over that of pure argon. Such mixtures have a thermal conductivity intermediate to the two pure gases. The ionization potential theory completely fails to explain why argon rich mixtures increase the rate of heat transfer. Since only 5 to $30 \%$ of the arc need be ionized, gas mixtures of less than 70 percent He contain no He ions. [Kim, 1986] If the He does not ionize, its ionization cannot be used to explain the more efficient heat transfer.

One may also ask why Argon-5\% $\mathrm{H}_{2}$ shielding gases also transfer heat more efficiently than pure argon. Using the simple mass diffusivity, thermal conductivity relationships presented above with a simple rule of mixtures, one would 
conclude that $\mathrm{Ar}-5 \% \mathrm{H}_{2}$ would have approximately a $25 \%$ greater thermal conductivity than pure argon. This cannot explain the greater rate of melting observed as this increase in thermal conductivity would result in only a 5\% increase in the amount of heat transformed to the anode. The explanation in this case is due to a third mechanism of heat conduction. In addition to the flow of the electric current and the thermal conductivity across the gas boundary layer, diatomic gases such as hydrogen also have an enhanced thermal conductivity due to dissociation of the gas in the plasma and recombination in the gas boundary layer. This enhancement of thermal conductivity in polyatomic gas plasmas is known as the reactive thermal conductivity. [Dunn, 1984] In fact, the thermal conductivity of $\mathrm{Ar}-5 \% \mathrm{H}_{2}$ is enhanced even more than 25\% over that of pure argon, due to this contribution of the reactive thermal conductivity.

\section{Droplet Formation in GMAW}

Photography of the gas metal arc process shows that the anode spot corona varies markedly with different shielding gases. With helium or carbon dioxide shielding, the anode spot is attached to the bottom of the pendant drop on the end of the electrode. The size of the spot does not change markedly as the current increases. With argon, the anode spot is more diffuse and grows noticably in size as the current increases. At sufficiently high currents (for example, above 23.0 amperes with $1.6 \mathrm{~mm}$ diameter steel electrodes) the anode spot fills the molten drop and begins to climb the cylindrical side walls of the electrode. Since most of the heat carried to the anode is carried by the welding current, the current in the anode spot causes the cylindrical side walls of the electrode to melt [Y.S. kim, 1989]. The end of the electrode forms a tapered geometry as shown in Figure 1 . Such a tapered electrode tip geometry does not develop in pure helium or pure carbon dioxide shielding gases.

The tapered tip creates a smaller diameter for attachment of the droplet to the electrode by surface tension forces [Y.S. Kim, 1989]. This produces smaller droplets than would be present in the absence of taper formation. The smaller droplets formed with argon shielding from a tapered electrode are seen clearly in Figure 2, as compared with helium and carbon dioxide shielding.

The formation of the taper occurs during gas metal arc welding of steel, aluminum and titanium, when using argon shielding gas [Y.S. Kim, 1989]. There are a number of consequences of this taper formation. Firstly, Y.S. Kim [1989] has shown that the melting rate of the electrode is controlled by heat transfer across the liquid-solid 


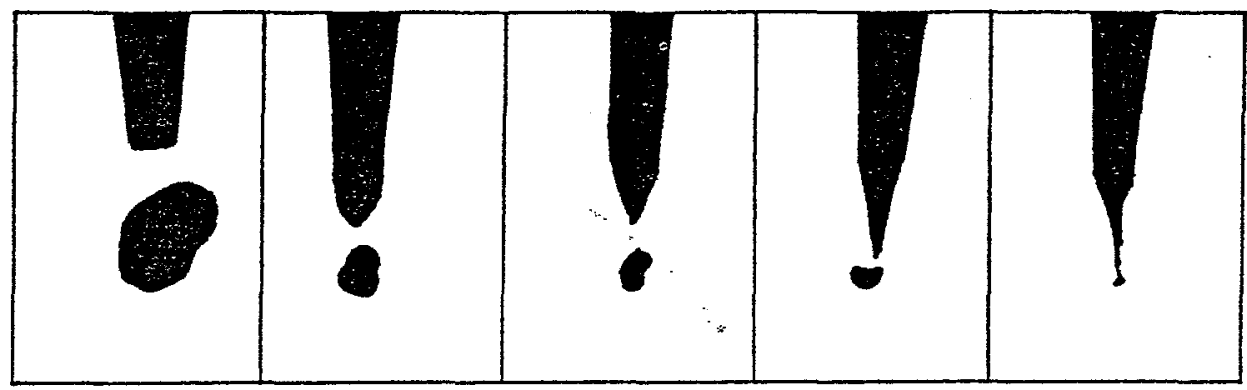

Fig. 1. Development of a taper as welding current increases when using argon shielding gas. amperes, (b) 237 amperes, (c) 253 amperes, (d) 281 amperes, and (e) 310 amperes.

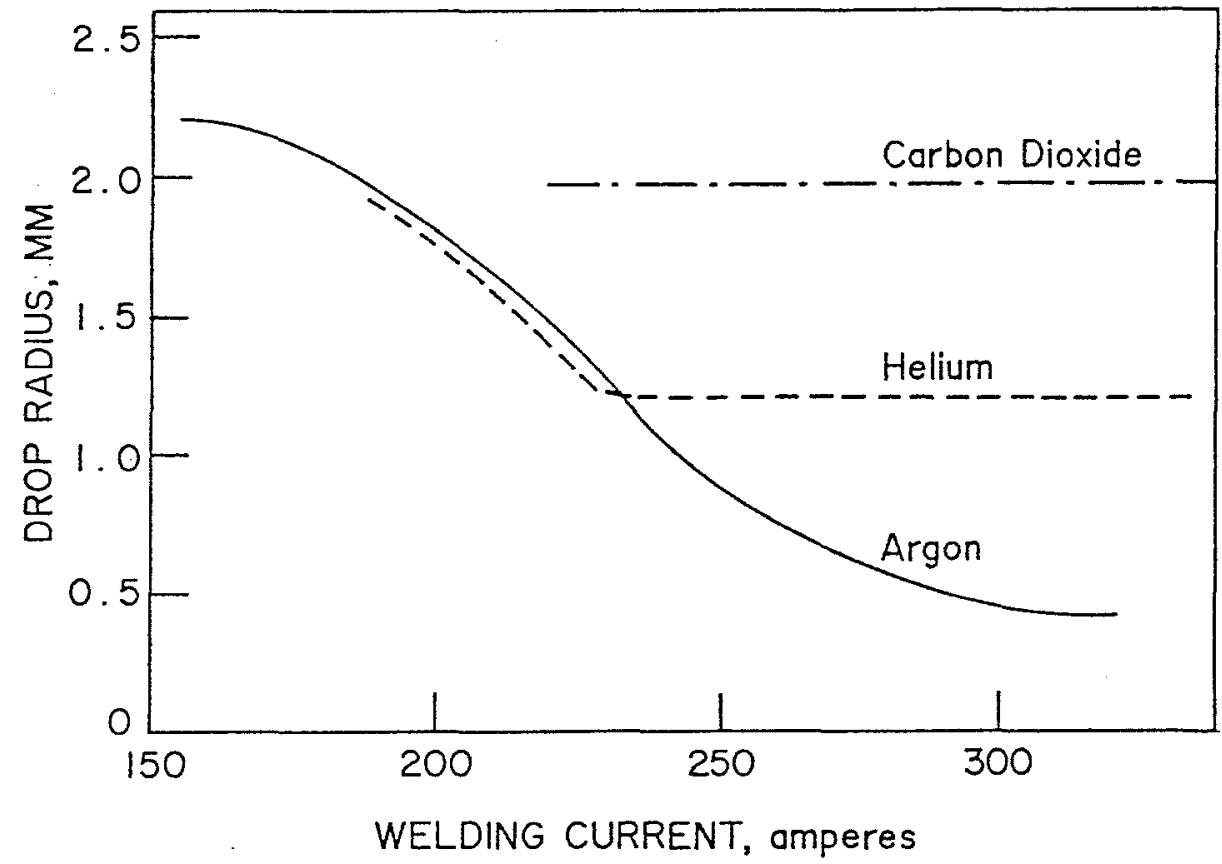

Fig. 2. Droplet size measured in three different shielding gases as a function of welding current for $1.6 \mathrm{~mm}$ diameter steel electrodes.

boundary between the drop and the solid electrode. Since the taper reduces the area of this boundary, taper

formation reduces the rate of melting of the electrode. At high currents, the melting rate in helium and carbon

dioxide is greater than in argon due to absence of the 
taper. Thus the greater melting rate in helium is not due to the higher ionization potential of this gas as is commonly assumed (see section 3 of this paper), but to a larger liquid-solid contact area in which heat is transferred to the solid electrode.

Secondly, Y.S. Kim has shown that the formation of a taper can reduce the range of one drop per pulse transfer in pulsed current GMAW. As the drops become smaller with argon shielding gas due to taper formation, the pulsing frequency must increase accordingly. By suppressing the formation of the taper, both large and small drops can be released from the end of the electrode during pulsed current welding. The largest drops are equal to the droplet size created at the low current portion of the pulse in the absence of the taper, while the smallest drops are equivalent to those produced during the peak of the current pulse. This greatly increases the range of frequencies which are permissible for one drop per pulse operation [Y.S. Kim and Eagar]. By addition of helium or carbon dioxide to the argon shielding gas, the anode spot is constricted and does not climb the cylindrical side walls of the electrode as readily. Thus taper formation is suppressed and the acceptable range of frequencies for pulsed current GMAW is broadened and the process becomes more easily controlled. This example illustrates how an understanding of the physics of droplet formation can aid the welding engineer in selection of the best shielding gas composition for pulsed current gas metal arc welding.

\section{Conclusion}

It is seen that an understanding of the arc physics can make a very complex process such as welding, appear much clearer. By understanding the process, it becomes easier to control and easier to modify our procedures to ensure that high quality, reliable joints are produced. With so many arc process variations and with an ever increasing number of materials to be joined, there is ample opportunity for many people to contribute to a better understanding of the physics of welding processes.

\section{Acknowledgements}

The author expresses his appreciation to the U.S. Department of Energy, the office of Naval Research and the National Science Foundation for support of the research presented herein. 


\section{References}

Dinulescu, A. and Pfender, E. (1980) Analysis of the Anode Boundary Layer of High Intensity Arcs. I. Appl. Phys., 51(6), 3149-3157.

Dunn, G.J. (1984) Metal Vapors in Gas Tungsten Welding Arcs. S.M. Thesis, Dept. of Materials Science and Engineering, MIT, Cambridge, MA.

Dunn, G.J. and:Eagar, T.W. (1986) Metal Vapors in Gas Tungsten Arcs: Part II. Theoretical Calculations of Transport Properties. Meta11. Trans., 17A(10), 1865-1871.

Ecker, G. (1980) Theoretical Aspects of the Vacuum Arc, in Vacuum Arcs, Theory and Application (ed. J.M. Lafferty), John Wiley, NY, pp. 228-320.

Giedt, W.H., Tallerico, L.N. and Fuerschbach, P.W. (1989) GTA Welding Efficiency: Calorimetric and Temperature Field Measurements. Welding $\mathrm{J}, 68(1), 28 \mathrm{~s}-32 \mathrm{~s}$.

Key, J.F., Chan, J.W. and McIlwain, M.E. (1983) Process Variable Influence on Arc Temperature Distribution. Welding J., $62(7), 179 \mathrm{~s}-184 \mathrm{~s}$.

Kim, E.W. (1986) Visible Light Emissions During Gas Tungsten ArC Welding. S.M. Thesis, Dept. of Materials Science and Engineering, MIT, Cambridge, MA.

Kim, Y.S. (1989) Metal Transfer in Gas Metal Arc Welding. Ph.D. Thesis, Dept. of Materials Science and Engineering, MIT, Cambridge, MA.

Kim, Y.S. and Eagar, T.W. Metal Transfer in Pulsed Current GMAW. Submitted to Welding J..

Quigley, M.B.C., Richards, P.H., Swift-Hook, D.T. and Gick, A.E.F. (1973) Heat Flow to the Workpiece From a TIG Welding Arc. I. Phys. D. - Appl. Phys, , 6, 2250-2258.

Savage, W.F., Strunck, S.S. and Ishikawa, Y. (1965) The Effect of Electrode Geometry in Gas Tungsten-Arc Welding. Welding $J_{1}, 44(11), 489 \mathrm{~s}-496 \mathrm{~s}$. 